\title{
Séminaires/Seminari 2015
}

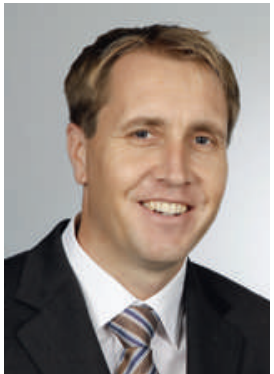

René Häller,

Directeur FMH Consulting Services

\section{Ouverture et reprise d'un cabinet médical}

Le séminaire est destiné aux médecins sur le point d'ouvrir un cabinet médical (individuel ou de groupe), de joindre un cabinet de groupe ou de reprendre un cabinet existant.

\section{Contenu}

- Business plan (préparation du plan de financement et crédit d'exploitation, financement par la banque)

- Aménagement (implantation, projet et concept d'aménagement, choix du mobilier, budget)

- Estimation d'un cabinet (inventaire et goodwill)

- Laboratoire

- Administration d'un cabinet médical

- Assurances

- Passage du statut de salarié à celui d'indépendant

- Fiscalité

\section{Sponsors}

Les coûts sont pris en charge par divers sponsors (voir www.fmhservices.ch).

\begin{tabular}{|c|c|c|}
\hline Dates & & \\
\hline K20 & $\begin{array}{l}\text { Jeudi } \\
5 \text { mars } 2015 \\
13 \mathrm{~h} 30-18 \mathrm{~h} 00\end{array}$ & $\begin{array}{l}\text { Lausanne } \\
\text { Mövenpick }\end{array}$ \\
\hline K21 & $\begin{array}{l}\text { Jeudi } \\
7 \text { mai } 2015 \\
\text { 13h30-18h00 }\end{array}$ & $\begin{array}{l}\text { Genève } \\
\text { Crowne Plaza }\end{array}$ \\
\hline K22 & $\begin{array}{l}\text { Jeudi } \\
3 \text { septembre } 2015 \\
17 \text { h00-21h30 }\end{array}$ & $\begin{array}{l}\text { Lausanne } \\
\text { World Trade } \\
\text { Center }\end{array}$ \\
\hline K23 & $\begin{array}{l}\text { Jeudi } \\
19 \text { novembre } 2015 \\
13 \mathrm{~h} 30-18 \mathrm{~h} 00\end{array}$ & $\begin{array}{l}\text { Genève } \\
\text { Crowne Plaza }\end{array}$ \\
\hline
\end{tabular}

\section{Remise d'un cabinet médical}

Le séminaire s'adresse aux médecins désirant remettre un cabinet médical. Idéalement 5-10 ans avant la remise prévue (pour des questions de taxation et de prévoyance).

Bien qu'environ $40 \%$ de tous les médecins ouvrent tôt ou tard leur propre cabinet médical individuel ou de groupe et qu'en plus de leur savoir-faire médical, ils aient besoin de connaissances en gestion d'entreprise, cette thématique est peu abordée lors des études. FMH Services, qui s'occupe de la gestion d'entreprise de ses membres, comble cette lacune en proposant, entre autres, des séminaires spécifiques qui réunissent chaque année quelque 750 médecins. Dans ces séminaires, gratuits pour la plupart, les participants peuvent acquérir les bases nécessaires pour ouvrir leur propre cabinet médical individuel ou de groupe. Ils obtiennent également des conseils sur la manière de collaborer avec les autorités ou avec des spécialistes tels que des experts-comptables, des conseillers financiers, en assurance ou en crédits bancaires, ainsi que des juristes, des architectes et autres. Ces différents thèmes sont abordés par des experts qualifiés de FMH Services.

\section{Contenu}

- Aspects juridiques (autour du contrat de remise/reprise)

- Estimation d'un cabinet (inventaire et goodwill)

- Assurances (prévoyance, assurances à l'intérieur et autour du cabinet)

- Conséquences fiscales d'une remise

\section{Sponsors}

Les coûts sont pris en charge par divers sponsors (voir www.fmhservices.ch).

\begin{tabular}{lll}
\hline Dates & & \\
\hline K24 & Jeudi & Lausanne \\
& 28 mai 2015 & World Trade \\
& 17h00-21h30 & Center \\
K25 & Jeudi & Genève \\
& 12 novembre 2015 & Crowne Plaza \\
& 17h00-21h30 &
\end{tabular}

\section{Cabinet de groupe}

Le séminaire s'adresse aux médecins en formation voulant exercer leur future activité en cabinet de groupe et aux libres praticiens qui souhaitent affilier leur cabinet individuel à un cabinet de groupe.

\section{Contenu}

- Stratégie (objectifs du cabinet de groupe; structure de l'offre de prestations)

- Entrepreneurs (composition de l'équipe; règles de conduite; participation financière et modèles de rémunération)

- Finances \& droit (assurances, prévoyance et patrimoine; forme juridique, finances et impôts)

- Lieu d'implantation \& immobilier (exigences locales; analyse de la situation concurrentielle; aménagement du cabinet et coûts)

- Ressources humaines (qualifications; lois, règlements et contrats)

- Direction \& organisation (structure et processus; tâches, responsabilités et compétences)
- Informatique \& administration (attentes en matière de système informatique pour le cabinet; évaluation)

- Rapport d'expérience (rapport de l'expérience d'un médecin, cofondateur d'un cabinet de groupe de la région)

\section{Sponsors}

Les coûts sont pris en charge par divers sponsors (voir www.fmhservices.ch).

\begin{tabular}{lll}
\hline Dates & & \\
\hline K85 & Jeudi & Genève \\
& 23 avril 2015 & Crowne Plaza \\
K86 & $\begin{array}{l}\text { 13h30-18h00 } \\
\text { Jeudi }\end{array}$ & Lausanne \\
& 24 septembre 2015 & World Trade \\
& 13h30-18h00 & Center \\
\hline
\end{tabular}

Constitution de patrimoine et optimisation fiscale

Le séminaire s'adresse aux médecins qui ne veulent pas laisser au hasard la planification de leur prévoyance et l'optimisation fiscale.

\section{Contenu}

Constitution de patrimoine et prévoyance

- Quel est le besoin éventuel de capital?

- Ma solution de prévoyance professionnelle est-elle suffisante ou y a-t-il des lacunes?

- Ma famille est-elle protégée?

- Les risques (en cas de soins ou de décès) sont-ils couverts?

- Une retraite anticipée est-elle possible pour moi?

Planification et optimisation fiscales

- Comment optimiser ma taxation de manière durable?

- La transformation de la forme juridique du cabinet est-elle judicieuse?

- Comment régler ma succession à long terme?

Coûts

100 CHF (y compris documents de cours et collations) 


\begin{tabular}{lll}
\hline Date & & \\
\hline K40 & Jeudi & Genève \\
& 30 avril 2015 & Crowne Plaza \\
& 17h00-20h00 & \\
\hline
\end{tabular}

\section{Inscription et information}

www.fmhservices.ch ou FMH Consulting Services, Cornelia Fuchs, Burghöhe 1, 6208 Oberkirch, Tél. 04192500 77, Fax 0419210586

\section{Remarque}

Les adresses des participants aux séminaires dont les coûts sont couverts en partie ou totalement par des sponsors sont communiquées aux sponsors concernés.

\section{Conditions d'annulation}

Un montant est perçu pour une absence ou une annulation. Il est de:

- 50 CHF par personne dans les 15 jours avant le début du séminaire;

- 100 CHF par personne dans les 7 jours avant le début du séminaire.
Apertura e rilevamento di uno studio medico

Il seminario è destinato ai medici in procinto di aprire o di rilevare uno studio medico.

\section{Contenuto}

- Business Plan (preparazione del piano di finanziamento e del credito d'esercizio, prestito bancario)

- Pianificazione (insediamento, progetto e pianificazione, scelta del mobilio, budget)

- Valutazione di uno studio medico (inventario e goodwill)

- Amministrazione di uno studio medico (interna allo studio, rapporti con la banca)

- Assicurazioni (tutte le assicurazioni necessarie interne ed esterne allo studio)

- Passaggio dallo stato di dipendente a quello di indipendente

- Fiscalità

\section{Sponsor}

Diversi sponsor si fanno carico delle spese (si rimanda al sito www.fmhservices.ch).

\begin{tabular}{lll}
\hline Date & & \\
\hline K50 & Giovedì & Chiasso \\
& $\begin{array}{l}\text { 16 aprile 2015 } \\
\text { dalle 14.00 alle 17.30 }\end{array}$ & FMH Consulting \\
K51 & $\begin{array}{l}\text { Giovedices } \\
\text { Chiasso }\end{array}$ \\
& 22 ottobre 2015 & FMH Consulting \\
& dalle 14.00 alle 17.30 & Services
\end{tabular}

\section{Studio medico associato}

Il seminario è destinato ai medici in formazione che desiderano esercitare la loro futura attività in uno studio medico associato. Liberi praticanti che auspicano affiliare o che hanno già affiliato il loro studio medico individuale ad uno associato.

\section{Contenuto}

- Strategia (obiettivi dello studio medico associato, struttura dell'offerta delle prestazioni)

- Imprenditori (composizione del gruppo di lavoro e regole di condotta, partecipazione finanziaria e metodi di remunerazione)

- Finanze e diritto (assicurazioni, previdenza e patrimonio, forma giuridica, finanze e fiscalità)

- Ubicazione e allestimento dello studio (esigenze dei locali e analisi della situazione concorrenziale, allestimento dello studio e costi)

- Risorse umane (qualifiche, regolamenti e contratti)

- Direzione e organizzazione (struttura e procedimenti, compiti, responsabilità e competenze)

- Informatica e amministrazione (aspettative dal sistema informatico per lo studio, valutazione dei sistemi d'informazione
Sponsor

Diversi sponsor si fanno carico delle spese (si rimanda al sito www.fmhservices.ch).

\begin{tabular}{lll}
\hline Date & & \\
\hline K88 & Giovedì & Chiasso \\
& $\begin{array}{l}7 \text { maggio 2015 } \\
\text { dalle 14.00 alle 18.00 }\end{array}$ & FMH Consulting \\
&
\end{tabular}

\section{Iscrizioni e informazioni}

www.fmhservices.ch o FMH Consulting Services, Cornelia Fuchs, Burghöhe 1, 6208 Oberkirch, Tél. 04192500 77, Fax 0419210586

\section{Osservazioni}

Gli indirizzi dei partecipanti ai seminari, i cui costi sono coperti in parte o completamente da degli sponsor, vengono comunicati agli sponsor interessati.

\section{Condizioni d'annullamento}

Un importo verrà rimborsato in caso di assenza o annullamento. Esso sarà di:

- 50 CHF per persona entro i 15 giorni prima dell'inizio del seminario;

- 100 CHF per persona entro i 7 giorni prima dell'inizio del seminario. 